\title{
Research Article \\ Bifurcation of a Cohen-Grossberg Neural Network with Discrete Delays
}

\author{
Qiming Liư ${ }^{1}$ and Wang Zheng ${ }^{2}$ \\ ${ }^{1}$ Institute of Applied Mathematics, Shijiazhuang Mechanical Engineering College, Shijiazhuang 050003, \\ China \\ ${ }^{2}$ Department of Orthopaedics, Hebei Provincial People's Hospital, Shijiazhuang 050051, China
}

Correspondence should be addressed to Qiming Liu, lqmmath@yahoo.com.cn

Received 7 January 2012; Accepted 31 January 2012

Academic Editor: Wing-Sum Cheung

Copyright (C) 2012 Q. Liu and W. Zheng. This is an open access article distributed under the Creative Commons Attribution License, which permits unrestricted use, distribution, and reproduction in any medium, provided the original work is properly cited.

\begin{abstract}
A simple Cohen-Grossberg neural network with discrete delays is investigated in this paper. The existence of local Hopf bifurcations is first considered by choosing the appropriate bifurcation parameter, and then explicit formulas are given to determine the direction of Hopf bifurcation and stability of the periodic solutions. Moreover, a set of sufficient conditions are given to guarantee the global Hopf bifurcation. Numerical simulations are given to illustrate the obtained results.
\end{abstract}

\section{Introduction}

In 1983, Cohen and Grossberg [1] proposed a kind of neural networks, which are now called Cohen-Grossberg neural networks. The networks have been successfully applied to signal processing, pattern recognition, optimization, and associative memories.

It is well known that the analysis of the dynamical behaviors is a necessary step for practical design of neural networks since their applications heavily depend on the dynamical behaviors. The research on dynamical behaviors of neural networks involves not only the dynamic analysis of equilibrium but also that of periodic solution, bifurcation, and chaos, especially, the periodic oscillatory behavior of the neural networks is of great interest in many applications $[2,3]$. Since periodic oscillatory can arise through the Hopf bifurcation in different system with or without time delays, it is very important to discuss the Hopf bifurcation of neural networks. Up to now, to the best of the author's knowledge, bifurcation of Hopfield neural networks has been discussed by many researchers [4-12], but only a few results on the bifurcation of Cohen-Grossberg neural networks have been obtained. Zhao discussed the bifurcation of a discrete-time Cohen-Grossberg neural network in [13] 
and the bifurcation of a continuous-time Cohen-Grossberg neural network with distributed delays in which kernel function is $\alpha \mathrm{e}^{-\alpha s}$ in [14]; Liu discussed the local Hopf bifurcation of the following Cohen-Grossberg neural network with discrete delays by regarding time delay as the bifurcation parameter in [15]:

$$
\begin{aligned}
& \dot{x}_{1}(t)=-a_{1}\left(x_{1}(t)\right)\left[b_{1}\left(x_{1}(t)\right)-d_{1} f_{2}\left(x_{2}\left(t-\tau_{2}\right)\right)\right], \\
& \dot{x}_{2}(t)=-a_{2}\left(x_{2}(t)\right)\left[b_{2}\left(x_{2}(t)\right)-d_{2} f_{1}\left(x_{1}\left(t-\tau_{1}\right)\right)\right],
\end{aligned}
$$

where $x_{i}(t)$ denotes the state variable associated with the $i$ th neuron; $a_{i}(\cdot)$ represents amplification function; $f_{i}(\cdot)$ denotes the signal function of the $i$ th neuron at time $t ; b_{i}(\cdot)$ is appropriately behaved function; $d_{i}(\cdot)$ is connection weight of the neural network; discrete delay $\tau_{i}$ corresponds to the finite speed of the axonal signal transmission at time $t, i=1,2$.

On the other hand, realistic modelling of neural networks inevitably depend on careful design and variation of the connection weight; in [16], Liu discussed the Neimark-Sacker bifurcation of a discrete-time version of the neural network (1.1) by regarding the connection weight as the bifurcation parameter. The objective of this paper is to discuss Hopf bifurcation of continuous-time system (1.1) by regarding the connection weight as the bifurcation parameter while time delays are fixed. The rest of this paper is organized as follows. Stability property, existence of Hopf bifurcation, and the stability of bifurcating periodic solutions for system (1.1) are obtained in Section 2. Global existence of Hopf bifurcation for system (1.1) is obtained in Section 3. An example is given in Section 4 to demonstrate the main results.

\section{Stability Analysis and Existence of Local Hopf Bifurcation}

Throughout this paper, we assume that

$\left(\mathrm{H}_{1}\right) b_{1}(0)=b_{2}(0)=0, f_{1}(0)=f_{2}(0)=0$;

$\left(\mathrm{H}_{2}\right)$ there exist constants $\underline{a}_{i}$, $\bar{a}_{i}$ such that $0<\underline{a}_{i} \leq a_{i}(\cdot) \leq \bar{a}_{i}$ for $i=1,2$;

$\left(\mathrm{H}_{3}\right)$ there exist constants $\underline{b}_{i}$ such that $b_{i}^{\prime}(\cdot) \geq \underline{b}_{i}>0$ for $i=1,2$.

Let $u_{1}(t)=x_{1}\left(t-\tau_{1}\right)$ and $u_{2}(t)=x_{2}(t)$; we transform system (1.1) into the following system

$$
\begin{aligned}
& \dot{u}_{1}(t)=-a_{1}\left(u_{1}(t)\right)\left[b_{1}\left(u_{1}(t)\right)-d_{1} f_{2}\left(u_{2}(t-\tau)\right)\right], \\
& \dot{u}_{2}(t)=-a_{2}\left(u_{2}(t)\right)\left[b_{2}\left(u_{2}(t)\right)-d_{2} f_{1}\left(u_{1}(t)\right)\right],
\end{aligned}
$$

in which $\tau=\tau_{1}+\tau_{2}$.

Obviously, system $(2.1)$ has an equilibrium $(0,0)$ under conditions $\left(\mathrm{H}_{1}\right)$ and $\left(\mathrm{H}_{2}\right)$.

The linearized system of system $(2.1)$ at $(0,0)$ is

$$
\begin{aligned}
& \dot{u}_{1}(t)=-a_{1}(0) b_{1}^{\prime}(0) u_{1}(t)+a_{1}(0) d_{1} f_{2}^{\prime}(0) u_{2}(t-\tau), \\
& \dot{u}_{2}(t)=-a_{2}(0) b_{2}^{\prime}(0) u_{2}(t)+a_{2}(0) d_{2} f_{1}^{\prime}(0) u_{1}(t) .
\end{aligned}
$$

The associated characteristic equation of system (2.2) is

$$
\lambda^{2}+a \lambda+b\left(c-d e^{-\lambda \tau}\right)=0,
$$


in which

$$
a=a_{1}(0) b_{1}^{\prime}(0)+a_{2}(0) b_{2}^{\prime}(0), \quad b=a_{1}(0) a_{2}(0), \quad c=b_{1}^{\prime}(0) b_{2}^{\prime}(0), \quad d=d_{1} d_{2} f_{1}^{\prime}(0) f_{2}^{\prime}(0)
$$

If $\tau=0$, it is easy to know that (2.3) has two roots with negative real parts when $d<c$. Equation (2.10) has at least one positive root when $d>c$.

If $\tau \neq 0$, let us assume that $\lambda=i \omega(\omega>0)$ is a root of (2.3); we have

$$
b c-\omega^{2}=b d \cos \omega \tau, \quad a \omega=-b d \sin \omega \tau .
$$

Adding up the squares of the corresponding sides of the above equation (2.5), we obtain

$$
\omega^{4}+k \omega^{2}+b^{2}\left(c^{2}-d^{2}\right)=0
$$

where $k=\left(a_{1}(0) b_{1}^{\prime}(0)\right)^{2}+\left(a_{2}(0) b_{2}^{\prime}(0)\right)^{2}$.

Denote $z=\omega^{2}$; then (2.6) becomes

$$
z^{2}+k z+b^{2}\left(c^{2}-d^{2}\right)=0
$$

Since $k>0$, (2.7) has no positive real roots when $|d| \leq c$. Equation (2.7) has a positive real roots when $|d|>c$, which means (2.3) has a pair of purely imaginary root for every $|d|>c$.

We have from (2.5) that

$$
\frac{a \omega}{\omega^{2}-b c}=\tan \omega \tau
$$

It has roots as follows $[4,12]$ :

$$
\omega_{j}^{+} \in\left[\frac{2(j-1) \pi}{\tau}, \frac{(2 j-1) \pi}{\tau}\right], \quad \omega_{j}^{-} \in\left[\frac{(2 j-1) \pi}{\tau}, \frac{(4 j-1) \pi}{2 \tau}\right], \quad j=1,2, \ldots
$$

From (2.5) and the discussion of (2.7), we obtain that

$$
d_{j}^{-}=-\frac{a \omega_{j}^{+}}{b \sin \omega_{j}^{+} \tau}<-b_{1}^{\prime}(0) b_{2}^{\prime}(0)<0, \quad d_{j}^{+}=-\frac{a \omega_{j}^{-}}{b \sin \omega_{j}^{-} \tau}>b_{1}^{\prime}(0) b_{2}^{\prime}(0)>0,
$$

that is, (2.3) has a pair of purely imaginary roots $\pm i \omega_{0}$ when $d=d_{j}^{ \pm}$.

On the other hand, we have

$$
\lambda^{\prime}(d)^{-1}=\frac{2 \lambda+a}{b e^{-\lambda \tau}}+\tau d=\left(\frac{2 \lambda+a}{\lambda^{2}+a \lambda+b c}+\tau\right) d
$$


thus we have

$$
\left.\operatorname{Re}\left(\lambda^{\prime}(d)^{-1}\right)\right|_{d=d_{j}^{ \pm}}=\left(\frac{a b c+a\left(\omega_{j}^{ \pm}\right)^{2}}{\left(b c-\omega_{j}^{ \pm}\right)^{2}+a^{2}\left(\omega_{j}^{ \pm}\right)^{2}}+\tau\right) d_{j}^{ \pm}
$$

which means

$$
\left.\operatorname{Re}\left(\lambda^{\prime}(d)^{-1}\right)\right|_{d=d_{j}^{+}}>0,\left.\quad \operatorname{Re}\left(\lambda^{\prime}(d)^{-1}\right)\right|_{d=d_{j}^{-}}<0,
$$

and note that at $d=b_{1}(0) b_{2}(0)=c,(2.3)$ has a root $\lambda(c)=0$, and $\lambda^{\prime}(c)^{-1}=(a / b+\tau) c>0$ according to (2.11).

From the above discusses and Hopf bifurcation theorem in [17] for functional differential equations, we have the following results.

Theorem 2.1. Under assumptions $\left(H_{1}\right)-\left(H_{3}\right)$, we have

(1) if $d_{1} d_{2} f_{1}^{\prime}(0) f_{2}^{\prime}(0) \in\left(d^{-}, b_{1}^{\prime}(0) b_{2}^{\prime}(0)\right)$, the equilibrium $(0,0)$ of system (1.1) is asymptotically stable;

(2) if $d_{1} d_{2} f_{1}^{\prime}(0) f_{2}^{\prime}(0) \notin\left[d^{-}, b_{1}^{\prime}(0) b_{2}^{\prime}(0)\right]$, the equilibrium $(0,0)$ of system (1.1) is unstable;

(3) $d_{j}^{ \pm}(j=0,1,2, \ldots)$ are Hopf bifurcation values for system (1.1),

where $d_{j}^{ \pm}$is given by $(2.10)$ and $d^{-}=\max _{j \geq 1}\left\{d_{j}^{-}\right\}$.

From discussion above, we obtain some conditions under which system (1.1) undergoes local Hopf bifurcation near $d=d_{j}^{ \pm}(j=1,2, \ldots)$. In addition, we can obtain explicit formulas for determining the properties of the Hopf bifurcation at critical $d=d_{j}^{ \pm}$.

Based on the normal form theory and the center manifold theorem [18], similar to discussion in [15], we can compute the following quantities:

$$
\begin{aligned}
C_{1}(0) & =\frac{i}{2 \omega_{j}^{\mp} \tau}\left(g_{20} g_{11}-2\left|g_{11}\right|^{2}-\frac{\left|g_{20}\right|^{2}}{3}\right)+\frac{g_{21}}{2}, \\
\mu_{2} & =-\frac{\operatorname{Re}\left\{C_{1}(0)\right\}}{\operatorname{Re}\left\{\lambda^{\prime}\left(d_{j}^{ \pm}\right)\right\}} \\
\beta_{2} & =2 \operatorname{Re}\left\{C_{1}(0)\right\}, \\
T_{2} & =-\frac{\operatorname{Im}\left\{C_{1}(0)\right\}+\mu_{2} \operatorname{Im}\left\{\lambda^{\prime}\left(d_{j}^{ \pm}\right)\right\}}{\tau \omega_{j}^{\mp}},
\end{aligned}
$$

where the explicit formulas for $g_{i j}$ in (2.14) can be obtained when we replace $\tau^{j}$ and $\omega_{0}$ in $g_{i j}$ by $\tau$ and $\omega_{j}^{\mp}$ in [15], respectively.

It is well known that $\mu_{2}$ determines the direction of the Hopf bifurcation and $\beta_{2}$ determines the stability of the bifurcating periodic solutions [18]. Since $\operatorname{Re}\left\{\lambda^{\prime}\left(d_{j}^{+}\right)\right\}>0$, 
we know if $\mu_{2}>0\left(\mu_{2}<0\right)$, then the Hopf bifurcation is supercritical(subcritical), the bifurcating periodic solutions exist for $d>d_{j}^{+}\left(d<d_{j}^{+}\right)$, and the bifurcating periodic solutions are stable(unstable), and due to $\operatorname{Re}\left\{\lambda^{\prime}\left(d_{j}^{-}\right)\right\}<0$, we know if $\mu_{2}<0\left(\mu_{2}>0\right)$, then the Hopf bifurcation is supercritical(subcritical), the bifurcating periodic solutions exist for $d<d_{j}^{-}\left(d>d_{j}^{-}\right)$, and the bifurcating periodic solutions are stable(unstable). $T_{2}$ determines the period of the bifurcating periodic solutions: the period increases (decreases) if $T_{2}>0\left(T_{2}<0\right)$.

Remark 2.2. The main results above coincide with the main results in [16].

\section{Global Existence of Hopf Bifurcation for the Model}

In this section, we mainly prove that the local Hopf bifurcation of (1.1) can be extended for large values by applying the global Hopf bifurcation theorem in [19].

$\left(\mathrm{H}_{4}\right)$ There exist constants $M_{i}>0$ such that $\left|f_{i}(\cdot)\right| \leq M_{i}$ and $f^{\prime}(\cdot) \geq 0, u_{i} f^{\prime \prime}\left(u_{i}\right)<0$ for $i=1,2$.

Remark 3.1. Assumption $\left(\mathrm{H}_{4}\right)$ is suitable, for example, $f_{i}\left(u_{i}\right)=\tanh u_{i}$ and $f_{i}\left(u_{i}\right)=(1-$ $\left.e^{-u_{i}}\right) /\left(1+e^{-u_{i}}\right)$ satisfy $\left(\mathrm{H}_{4}\right)$.

Theorem 3.2. Under assumptions $\left(H_{1}\right)-\left(H_{4}\right)$ and either $\left(d_{1}, f_{1}(0), f_{2}(0)\right)$ or $\left(d_{2}, f_{1}(0), f_{2}(0)\right)$ is fixed, system (1.1) has at least a periodic solution for $d<d_{j}^{-}$.

Proof. First, we can prove that system (1.1) has a unique equilibrium $(0,0)$ under conditions $\left(\mathrm{H}_{1}\right)-\left(\mathrm{H}_{4}\right)$ when $d<d^{-}$.

The equilibria of system (1.1) satisfy the following equation according to condition $\left(\mathrm{H}_{2}\right)$ :

$$
\begin{aligned}
& b_{1}\left(u_{1}\right)-d_{1} f_{2}\left(u_{2}\right)=0, \\
& b_{2}\left(u_{2}\right)-d_{2} f_{1}\left(u_{1}\right)=0 .
\end{aligned}
$$

From the first equation of (3.1), we have $u_{1}=b_{1}^{-}\left(d_{1} f_{2}\left(u_{2}\right)\right)$; substitute it into the second equation of (3.1); we obtain

$$
b_{2}\left(u_{2}\right)-d_{2} f_{1}\left(b_{1}^{-}\left(d_{1} f_{2}\left(u_{2}\right)\right)\right)=0 .
$$

Denote

$$
g\left(u_{2}\right)=b_{2}\left(u_{2}\right)-d_{2} f_{1}\left(b_{1}^{-}\left(d_{1} f_{2}\left(u_{2}\right)\right)\right)
$$

and we have

$$
g^{\prime}\left(u_{2}\right)=b_{2}^{\prime}\left(u_{2}\right)\left[1-d_{1} d_{2} \frac{f_{1}^{\prime}\left(b_{1}^{-}\left(d_{1} f_{2}\left(u_{2}\right)\right)\right) f_{2}^{\prime}\left(u_{2}\right)}{b_{2}^{\prime}\left(u_{2}\right) b_{1}^{\prime}\left(d_{1} f_{2}\left(u_{2}\right)\right)}\right] .
$$

When $d_{1} d_{2}<0$, obviously, $g^{\prime}\left(u_{2}\right)>0$ due to $f_{i}^{\prime}(\cdot)>0$ according to $\left(\mathrm{H}_{4}\right)$. When $d_{1} d_{2}>0$, we obtain that $f_{i}^{\prime}(0)=\max _{u_{i} \in R} f_{i}^{\prime}\left(u_{i}\right), i=1,2$, due to $u_{i} f_{i}^{\prime \prime}\left(u_{i}\right)<0$ according to $\left(\mathrm{H}_{4}\right)$, combined 
with $d=d_{1} d_{2} f_{1}^{\prime}(0) f_{2}^{\prime}(0)<d^{-}<0$ and condition $\left(\mathrm{H}_{3}\right)$; we obtain $d_{1} d_{2} f_{1}^{\prime}\left(b_{1}^{-}\left(d_{1} f_{2}\left(u_{2}\right)\right)\right) f_{2}^{\prime}\left(u_{2}\right)<$ $d_{1}^{-}<0$; consequently, $g^{\prime}\left(u_{2}\right)>0$. Hence $g\left(u_{2}\right)$ is an increasing bijective function, since $g_{2}(0)=$ $0, u_{2}=0$ is a unique solution of (3.3), and (3.1) has a unique solution $(0,0)$, that is, $(0,0)$ is a unique equilibrium of system (1.1) when $d<d^{-}$.

Second, let us prove the global existence of Hopf bifurcation of system (1.1). Following the work of $\mathrm{Wu}[19]$, we make the following definitions.

Denote the equivalent system (2.1) of system (1.1) as follows:

$$
\dot{u}=F\left(u_{t}(\theta), \tau\right)
$$

in which $u=\left(u_{1}, u_{2}\right), u_{t}(\theta)=u(t+\theta) \in C\left([-\tau, 0], R^{2}\right)$.

And denote

$$
X=\mathrm{C}\left([-\tau, 0], R^{2}\right), \quad \Sigma=\mathrm{cl}\left\{(u(t), d, p) \in X \times R \times R^{+}\right\},
$$

where $u(t)$ is a $p$-periodic solution of $(2.1)$. Let $\ell\left(0, d_{j}^{-}, 2 \pi / \omega_{j}^{+}\right)$denote the connected component through $\left(0, d_{j}^{-}, 2 \pi / \omega_{j}^{+}\right)$in $\Sigma$, where $d_{j}^{-}$and $\omega_{j}^{+}$are given by (2.9) and (2.10), respectively. From Theorem 2.1, we know that $\left(0, d_{j}^{-}, 2 \pi / \omega_{j}^{+}\right)$is nonempty, and all centers are isolated center. At fact, sets of centers are

$$
\left\{\left(0, d_{j}^{-}, \frac{2 \pi}{\omega_{j}^{+}}\right), j=1,2, \ldots\right\}
$$

The characteristic function

$$
\Delta(0, d, p)(\lambda)=\lambda^{2}+a \lambda+b\left(c-d e^{-\lambda \tau}\right)
$$

is continuous in $(d, p, \lambda) \in R \times R \times R \times C$. Theorem 2.1 above ensures that there exist $\varepsilon>0, \epsilon>0$ and a smooth curve $\lambda:\left(d_{j}^{-}-\delta, d_{j}^{-}+\delta\right) \rightarrow C$ such that $\Delta(\lambda(d))=0,\left|\lambda(d)-i \omega_{j}^{+}\right|<\epsilon$ for all $d \in\left[d_{j}^{-}-\delta, d_{j}^{-}+\delta\right]$, and $\lambda\left(d_{j}^{-}\right)=i \omega_{j}^{+},\left.\operatorname{Re}\left(\lambda^{\prime}(d)^{-1}\right)\right|_{d=d_{j}^{-}}<0$. Define $\Omega_{\varepsilon}=\{(u, p): 0<u<$ $\epsilon,\left|p-2 \pi / \omega_{j}^{+}\right|<\varepsilon$. It is not difficult to show that $\Delta(0, d, p)(u+i 2 \pi / p)=0$ on $\left[d_{j}^{-}-\delta, d_{j}^{-}+\delta\right] \times \partial \Omega_{\epsilon}$ if and only if $d=d_{j}^{-}, u=0, p=2 \pi / \omega_{j}^{+}$.

Moreover, if we define

$$
H^{ \pm}\left(0, d_{j}^{-}, \frac{2 \pi}{\omega_{j}^{+}}\right)(u, p)=\Delta\left(0, d_{j}^{-} \pm \delta, p\right)\left(u+i \frac{2 \pi}{p}\right)
$$

and we can compute the crossing number of every isolated center $\left(0, d_{j}^{-}, 2 \pi /\left(\omega_{j}^{+}\right)\right)$as follows:

$$
\gamma\left(0, d_{j}^{-}, \frac{2 \pi}{\omega_{j}^{+}}\right)=\operatorname{deg}_{B}\left(H^{-}\left(0, d_{j}^{-}, \frac{2 \pi}{\omega_{j}^{+}}\right), \Omega_{\varepsilon}\right)-\operatorname{deg}_{B}\left(H^{+}\left(0, d_{j}^{-}, \frac{2 \pi}{\omega_{j}^{+}}\right), \Omega_{\varepsilon}\right)=-1,
$$

hence we can obtain from Theorem 3.3 in [19] that the connected component $\ell\left(0, d_{j}^{-}, 2 \pi\right.$ / $\left.\left(\omega_{j}^{+}\right)\right)$in $\Sigma$ is unbounded. 
On the other hand, we can prove that all nontrivial periodic solutions of system (2.1) are uniformly bounded.

Let $V(t)=\sqrt{u_{1}^{2}+u_{2}^{2}}$, we have

$$
\begin{aligned}
\dot{V}(t)= & \frac{1}{V(t)}\left[-a_{1}\left(u_{1}(t)\right) b_{1}\left(u_{1}(t)\right) u_{1}(t)+d_{1} f_{2}\left(u_{2}(t-\tau)\right) u_{1}(t)\right. \\
& \left.-a_{2}\left(u_{2}(t)\right) b_{2}\left(u_{2}(t)\right) u_{2}(t)+d_{2} f_{1}\left(u_{1}(t) u_{2}(t)\right)\right] \\
\leq & \frac{1}{V(t)}\left[-\underline{a}_{1} b_{1} u_{1}(t)+\left|d_{1}\right| M_{2} u_{1}(t)-\underline{a}_{2} b_{2} u_{2}(t)+\left|d_{2}\right| M_{1} u_{2}(t)\right] \\
\leq & \frac{1}{V(t)}\left[-k_{1} V^{2}(t)+\sqrt{2} k_{2} V(t)\right] \\
\leq & -k_{1} V(t)+\sqrt{2} k_{2}
\end{aligned}
$$

where $k_{1}=\max \left\{\underline{a}_{1} b_{1}, \underline{a}_{2} b_{2}\right\}$ and $k_{2}=\max \left\{\left|d_{1}\right| M_{1},\left|d_{2}\right| M_{2}\right\}$. It follows that

$$
V(t) \leq k+V(0) e^{-k t} \stackrel{t \rightarrow+\infty}{\longrightarrow} k, \quad k=\frac{\sqrt{2} k_{2}}{k_{1}}
$$

Hence, all nontrivial periodic solutions of system (2.1) are uniformly bounded.

At the same time, we can prove that the period $p$ of a periodic solution of system (2.1) with $d<d^{-}$on $\ell\left(0, d_{j}^{-}, 2 \pi / \omega_{j}^{+}\right)$is also uniformly bounded. In fact, system has no nontrivial $\tau$-periodic solution. For a contradiction, suppose that system (2.1) has a nontrivial $\tau$-periodic solution, then the following differential equation also has a nontrivial $\tau$-periodic solution

$$
\begin{aligned}
& \dot{u}_{1}(t)=-a_{1}\left(u_{1}(t)\right)\left[b_{1}\left(u_{1}(t)\right)-d_{1} f_{2}\left(u_{2}(t)\right)\right]=P\left(u_{1}, u_{2}\right), \\
& \dot{u}_{2}(t)=-a_{2}\left(u_{2}(t)\right)\left[b_{2}\left(u_{2}(t)\right)-d_{2} f_{1}\left(u_{1}(t)\right)\right]=Q\left(u_{1}, u_{2}\right) .
\end{aligned}
$$

On the other hand, let

$$
\begin{gathered}
B\left(u_{1}, u_{2}\right)=\frac{1}{a_{1}\left(u_{1}\right) a_{2}\left(u_{2}\right)} \\
\frac{\partial(B P)}{\partial u_{1}}+\frac{\partial(B Q)}{\partial u_{2}}=-\left[\frac{b_{1}^{\prime}\left(u_{1}\right)}{a_{2}\left(u_{2}\right)}+\frac{b_{2}^{\prime}\left(u_{2}\right)}{a_{1}\left(u_{1}\right)}\right] \leq-\left[\frac{b_{1}}{\bar{a}_{2}}+\frac{b_{2}}{\bar{a}_{2}}\right]<0 .
\end{gathered}
$$

Thus, we conclude that system (2.6) has no $\tau$-periodic solution according to Bendixson-Dulac criterion. Consequently, system (2.1) has no $\tau / n$-periodic solution for positive integer $n \geq 1$. From definition of $\omega_{j}^{+}$, we have

$$
\frac{\tau}{m}<p<\frac{2 \pi}{\omega_{1}^{+}}
$$

where $m$ is a positive integer. Therefore, the projection of $\ell\left(0, d_{j}^{-}, 2 \pi / \omega_{j}^{+}\right)$on $p$-space is bounded. 
Hence, we obtain that the projection $\ell\left(0, d_{j}^{-}, 2 \pi / \omega_{j}^{+}\right)$on $d$-space must be unbounded. As a result, for any $d<d_{j}^{-}$, system (2.1) has at least a periodic solution with a period in $\left(\tau / m, 2 \pi / \omega_{1}^{+}\right)$and the proof of Theorem 3.2 is complete.

Remark 3.3. The discussions of bifurcations of system (1.1) at $d=b_{1}^{\prime}(0) b_{2}^{\prime}(0)$ are a very cockamamie case. It is difficult to obtain good conditions to guarantee the global existence of periodic solutions of system (1.1), but if $a_{1}\left(x_{1}(t)\right)=a_{2}\left(x_{2}(t)\right)=1, b_{1}\left(t, x_{1}(t)\right)=\mu_{1} x_{1}(t)$, and $b_{2}\left(t, x_{2}(t)\right)=\mu_{2}(t) x_{2}(t)$, system (1.1) reduces to the following Hopfield neural network:

$$
\begin{aligned}
& \dot{x}_{1}(t)=-\mu_{1} x_{1}(t)+d_{1} f_{2}\left(x_{2}\left(t-\tau_{2}\right)\right), \\
& \dot{x}_{2}(t)=-\mu_{1} x_{1}(t)+d_{2} f_{1}\left(x_{1}\left(t-\tau_{1}\right)\right) .
\end{aligned}
$$

A supercritical pitchfork of system (3.16) can occur at $d=\mu_{1} \mu_{2}$ under assumptions $\left(\mathrm{H}_{1}\right)$ $\left(\mathrm{H}_{4}\right)$ [11], and the periodic solutions of system (2.2) are also globally existent [12]. Of course, Theorems 2.2 and Theorem 3.1 for (3.16) in [12] are special cases of Theorems 2.1 and 3.2 in this paper.

\section{A Numerical Example}

Consider the following Cohen-Grossberg neural network with discrete delays:

$$
\begin{aligned}
& \dot{x}_{1}(t)=-\left(6+\cos \left(x_{1}(t)\right)\right)\left[x_{1}(t)-d_{1} \tanh \left(x_{2}(t-2)\right)\right], \\
& \dot{x}_{2}(t)=-4\left[2 x_{2}(t)+\tanh \left(x_{1}(t-1)\right)\right] .
\end{aligned}
$$

Since $f^{\prime}(0)=1, b_{1}^{\prime}(0)=1, b_{2}^{\prime}(0)=2, a_{1}(0)=7$, and $a_{2}(0)=4, d_{2}=1$, using MATLAB 7.0, we can obtain that

$$
\begin{aligned}
b_{1}^{\prime}(0) b_{2}^{\prime}(0) & =2, \\
d & =d_{1} d_{2} f_{1}^{\prime}(0) f_{2}^{\prime}(0)=d_{1}, \\
\omega_{1}^{+} & \approx 0.9618, \quad d_{1}^{-} \approx-2.0334, \\
\omega_{2}^{+} & \approx 2.8952, \quad d_{2}^{-} \approx-2.3023, \\
\omega_{3}^{+} & \approx 4.8522, \quad d_{3}^{-} \approx-2.8461, \ldots, \\
d^{-} & =d_{1}^{-} \approx 2.0334 .
\end{aligned}
$$

Moreover, due to $f^{\prime \prime}(0)=0, f^{\prime \prime \prime}(0)=2, a_{1}^{\prime}(0)=a_{2}^{\prime}(0)=a_{2}^{\prime \prime}(0)=0, a_{1}^{\prime \prime}(0)=1$, and $b_{1}^{\prime}(0)=b_{2}^{\prime}(0)=0$, it is easy to know that $g_{20}=g_{11}=g_{02}=0$ and

$$
g_{21}=3 \bar{D}\left[14 d_{1} \bar{\rho} \rho^{2} e^{-i 3 \omega_{j}^{+}}+8\right]
$$

Case 1. Let $d_{1}=-1.8$. Note that $d=-1.8 \in(-2.0334,2)$, system (4.1) satisfies Theorem 2.1, and the equilibrium $(0,0)$ of system $(4.1)$ is asymptotically stable. Figure 1 shows the dynamic behaviors of system (4.1) with initial condition $(0.1,0.2)$. 


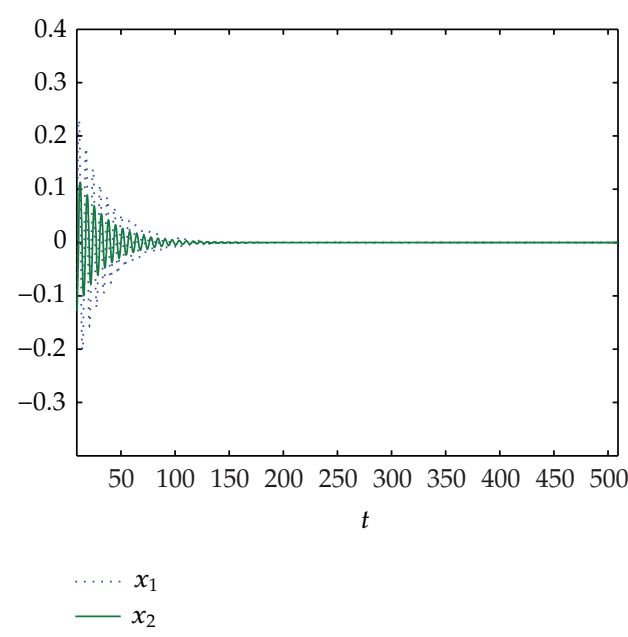

(a)

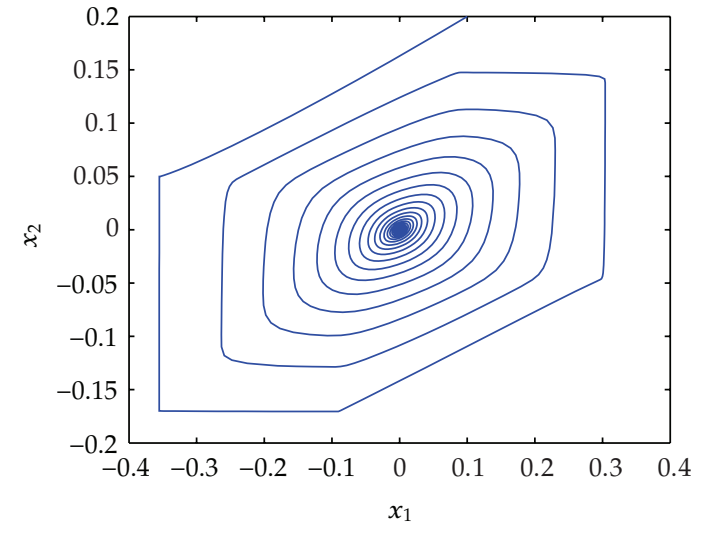

(b)

Figure 1: (a) Phase plot in space $\left(x_{1}, x_{2}\right)$ for system (4.1) with $d_{1}=-1.8$. (b) Phase plot in space $\left(x_{1}, x_{2}\right)$ for system (4.1) with $d_{1}=-1.8$.

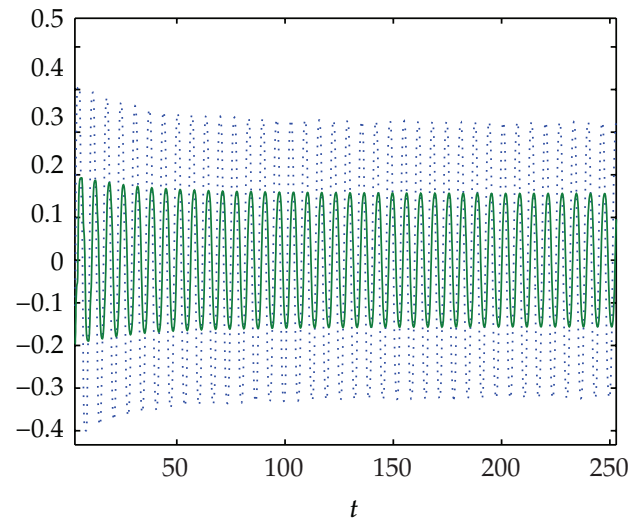

$\cdots \cdot x_{1}$

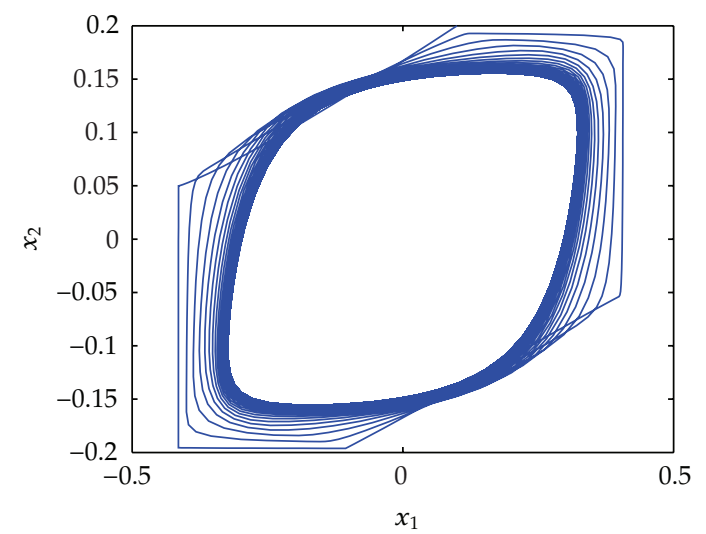

(a)

(b)

Figure 2: (a) Phase plot in space $\left(x_{1}, x_{2}\right)$ for system (4.1) with $d_{1}=-2.1$. (b) Phase plot in space $\left(x_{1}, x_{2}\right)$ for system (4.1) with $d_{1}=-2.1$.

Case 2. Let $d_{1}=-2.1$ and -3.0 , respectively. Note that $d=-2.1,-3.0<d^{-}=-2.0334$, the equilibrium $(0,0)$ loses its stability, and Hopf bifurcations occurs, that is, periodic solutions bifurcate from $(0,0)$ according to Theorem 3.2. Furthermore, we can from (4.3) obtain that $g_{21}\left(d_{1}^{-}\right)=-70.7796-1.8445 i, g_{21}\left(d_{2}^{-}\right)=-9.7399-1.1614 i, g_{21}\left(d_{3}^{-}\right)=-5.6912-0.9305 i, \ldots ;$ so, $\mu_{2}<0$ for $d_{j}^{-}, j=1,2,3$. Hence, the periodic solutions are stable for $d_{1}=-2.1,-3.0$. Figures 2 and 3 show the dynamic behaviors of system (4.1) with initial condition $(0.1,0.2)$. 


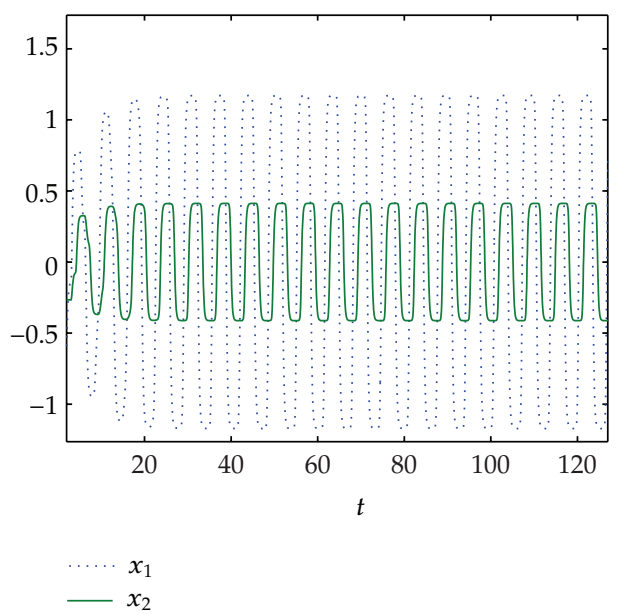

(a)

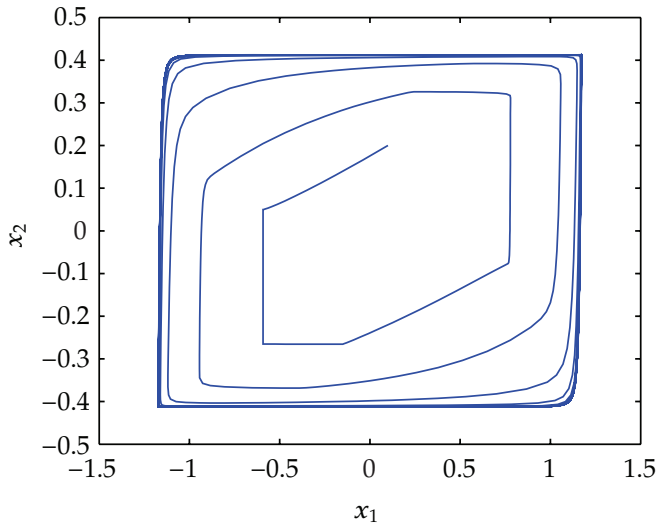

(b)

Figure 3: (a) Phase plot in space $\left(x_{1}, x_{2}\right)$ for system (4.1) with $d_{1}=-3.0$. (b) Phase plot in space $\left(x_{1}, x_{2}\right)$ for system (4.1) with $d_{1}=-3.0$.

\section{References}

[1] M. A. Cohen and S. Grossberg, "Absolute stability of global pattern formation and parallel memory storage by competitive neural networks," IEEE Transactions on Systems, Man, and Cybernetics, vol. 13, no. 5, pp. 815-826, 1983.

[2] S. Townley, A. Ilchmann, M. G. Weiß et al., "Existence and learning of oscillations in recurrent neural networks," IEEE Transactions on Neural Networks, vol. 11, no. 1, pp. 205-214, 2000.

[3] Z. Huang and Y. Xia, "Exponential periodic attractor of impulsive BAM networks with finite distributed delays," Chaos, Solitons and Fractals, vol. 39, no. 1, pp. 373-384, 2009.

[4] J. Wei and S. Ruan, "Stability and bifurcation in a neural network model with two delays," Physica D, vol. 130, no. 3-4, pp. 255-272, 1999.

[5] J. Cao and M. Xiao, "Stability and Hopf bifurcation in a simplified BAM neural network with two time delays," IEEE Transactions on Neural Networks, vol. 18, no. 2, pp. 416-430, 2007.

[6] S. Guo, L. Huang, and L. Wang, "Linear stability and Hopf bifurcation in a two-neuron network with three delays," International Journal of Bifurcation and Chaos in Applied Sciences and Engineering, vol. 14, no. 8, pp. 2799-2810, 2004.

[7] C. Huang, L. Huang, J. Feng, M. Nai, and Y. He, "Hopf bifurcation analysis of a two-neuron network with four delays," Chaos, Solitons and Fractals, vol. 34, no. 3, pp. 795-812, 2007.

[8] X. Zhou, Y. Wu, Y. Li, and X. Yao, "Stability and Hopf bifurcation analysis on a two-neuron network with discrete and distributed delays," Chaos, Solitons and Fractals, vol. 40, no. 3, pp. 1493-1505, 2009.

[9] Y. Yang and J. Ye, "Stability and bifurcation in a simplified five-neuron BAM neural network with delays," Chaos, Solitons and Fractals, vol. 42, no. 4, pp. 2357-2363, 2009.

[10] Y. Song, M. Han, and J. Wei, "Stability and Hopf bifurcation analysis on a simplified BAM neural network with delays," Physica D, vol. 200, no. 3-4, pp. 185-204, 2005.

[11] J. J. Wei, C. R. Zhang, and X. L. Li, "Bifurcation in a two-dimensional neural network model with delay," Applied Mathematics and Mechanics, vol. 26, no. 2, pp. 193-200, 2005 (Chinese).

[12] J. Wei, M. G. Velarde, and V. A. Makarov, "Oscillatory phenomena and stability of periodic solutions in a simple neural network with delay," Nonlinear Phenomena in Complex Systems, vol. 5, no. 4, pp. 407-417, 2002.

[13] H. Zhao and L. Wang, "Stability and bifurcation for discrete-time Cohen-Grossberg neural network," Applied Mathematics and Computation, vol. 179, no. 2, pp. 787-798, 2006.

[14] H. Zhao and L. Wang, "Hopf bifurcation in Cohen-Grossberg neural network with distributed delays," Nonlinear Analysis. Real World Applications, vol. 8, no. 1, pp. 73-89, 2007. 
[15] Q. Liu and R. Xu, "Stability and bifurcation of a Cohen-Grossberg neural network with discrete delays," Applied Mathematics and Computation, vol. 218, no. 6, pp. 2850-2862, 2011.

[16] Q. Liu, R. Xu, and Z. Wang, "Stability and bifurcation of a class of discrete-time Cohen-Grossberg neural networks with delays," Discrete Dynamics in Nature and Society, Article ID 403873, 14 pages, 2011.

[17] J. K. Hale, Theory of Functional Differential Equations, Springer, New York, NY, USA, 1977.

[18] B. D. Hassard, N. D. Kazarinoff, and Y. H. Wan, Theory and Applications of Hopf Bifurcation, vol. 41, Cambridge University Press, Cambridge, UK, 1981.

[19] J. Wu, "Symmetric functional-differential equations and neural networks with memory," Transactions of the American Mathematical Society, vol. 350, no. 12, pp. 4799-4838, 1998. 


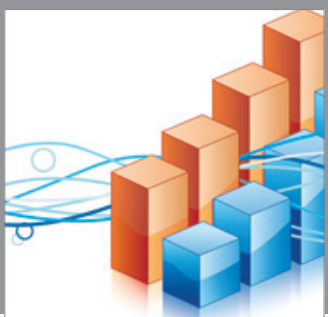

Advances in

Operations Research

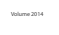

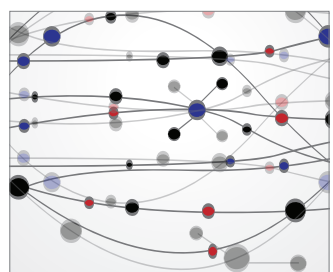

\section{The Scientific} World Journal
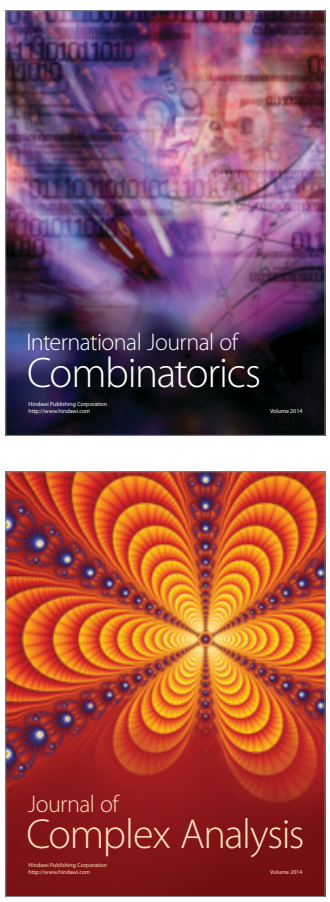

International Journal of

Mathematics and

Mathematical

Sciences
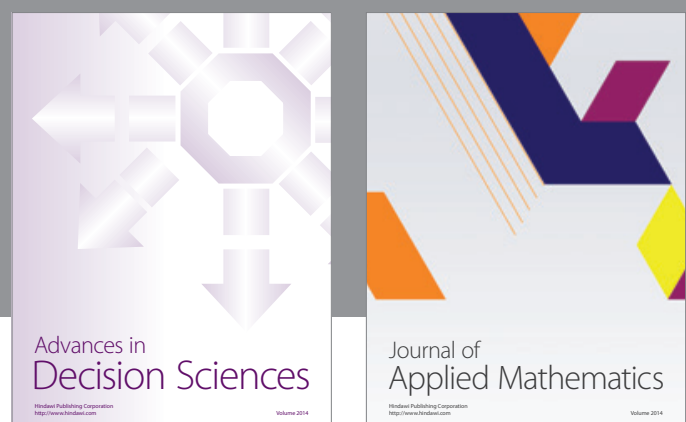

Journal of

Applied Mathematics
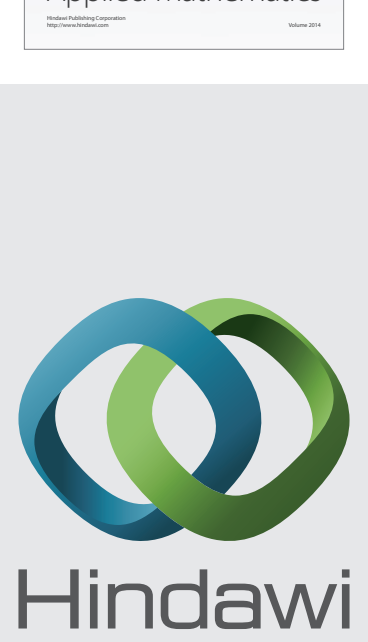

Submit your manuscripts at http://www.hindawi.com
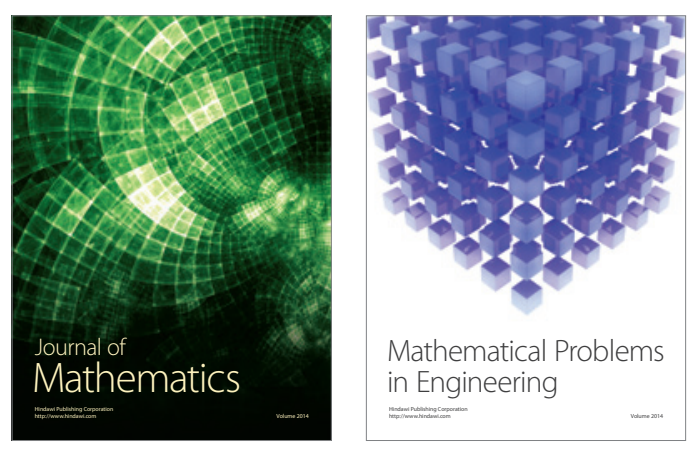

Mathematical Problems in Engineering
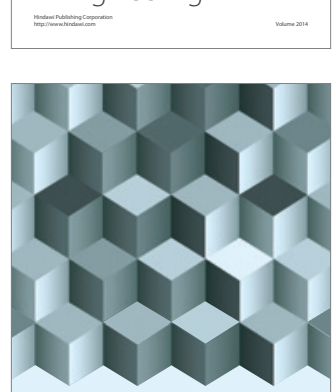

Journal of

Function Spaces
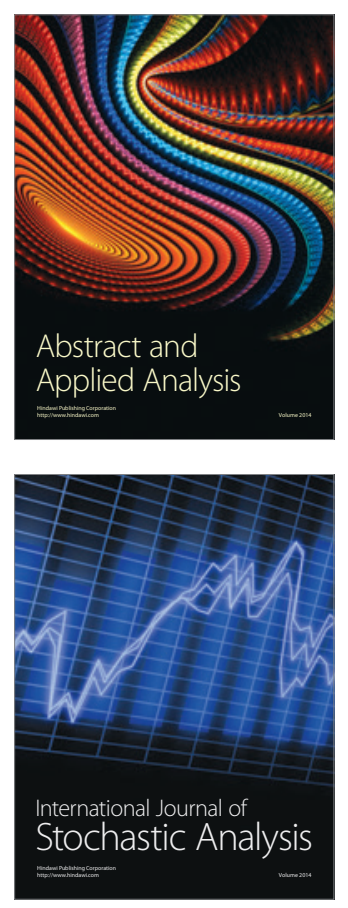

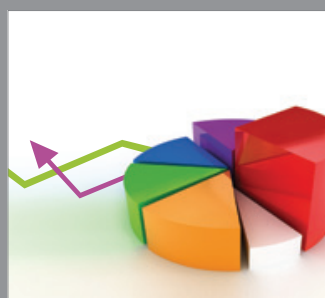

ournal of

Probability and Statistics

Promensencen
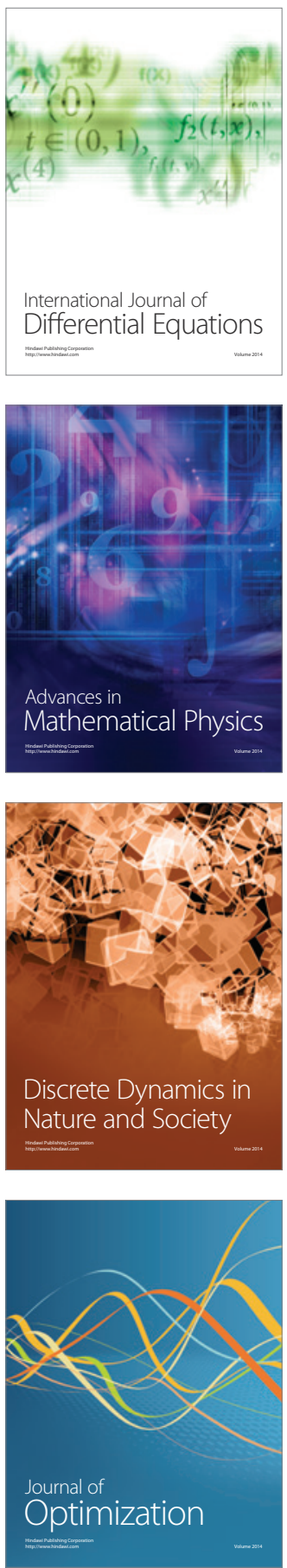